Dermatologische Zeitschrift. 1925;43:I-IV

\title{
Contents, Vol. 43, 1925
}

Inhaltsverzeichnis.

Originalarbeiten. Seite

Behrmann, K., Über die Zweckmäßigkeit von Paralleluntersuchungen nach der Wassermannschen, Bruckschen und Meinickeschen Reaktion (M. T. R.) bei der Serodiagnose der Syphilis 29

Boas, Ha raid, Untersuchungen über die Flockungsreaktion von Kahn .... $\quad 140$

Bruck, C, Ein Fall von enormer Wismutüberdosierung ohne schädliche Folgen 178

Diet el, Friedr., Ein Fall von Hautblutungen und Geschwüren bei Gonorrhöe $\quad 300$ Hoffmann, Erich, Über das knollentreibende Fibrosarkom der Haut (Dermatofibrosarkoma protuberans) 1 Ein Merkblatt über die Bedeutung der Lumbalpunktion bei Syphilitischen nebst Bemerkungen über moderne Syphilistherapie und Mesurol 296

Herxheimer, K. un,d H. Martin, Beitrag zur Klinik der der Tuberkulide 127

Kirschenblatt, D., Ein Fall von ungewöhnlicher exsudativer polymorpher Dermatose 43

Kottmaier, Elsa, Die Linsersche Varizenbehandlung und ihr Wert für die Dermatologie Í86

Lehner, E., Beitrag zur Pathogenese des Herpes zoster . . 33 Martin, H., siehe Herxheimer, K. Mietling, Hans, Liquorbefunde bei behandelter Syphilis . 180 Mühlhoff, Paul, Zur Reargonbehandlung der Gonorrhöe . 305 Neergaard, K. von, Ein Beitrag zur Theorie der Silbersalzwirkung 266

- und R. S c h är e r, Die Benutzung quellungsfähiger Kolloide in Verbindung mit einer Silber-Depôt-Behandlung der weiblichen Gonorrhöe 149

Pinkus, Felix, Zur Kenntnis der menschlichen Schweißdrüsen 253

Schärer, R., siehe Neergaard, K. von.

Ir Inhaltsverzeîchnis.

Seito Stühmer, A., Bilharziaerkrankung der Blase. (Hierzu Tafel I-П) 132

Weyrauch, Friedr., Zu $\gamma$ Beurteilung der Spätflockungen, insbesondere bei der Dold-Reaktion 40 
Wirz, Franz, Zur Polymorphie der Salvarsanexan theme.

(Lupus-erythematodes-ähnlicher Fall) 260

Zum 70. Geburtstag von Hofrat Professor Dr. G. R i e h 1. Von

Erich Hoffmann-Bonn a. Rh 252

Gesellschafísberichte.

Berliner Dermatologische Gesellschaft. Sitzung vom 18. VII.

192451

Frühjahrsversammlung der Vereinigung rhein. ' westf. Derma-

tologen in Köln am 18. V. $1924 \quad 68$

Kiewer Gesellschaft für Haut · und Geschlechtskrankheiten.

Sitzungen vom 4. IV., 4. V., 7. X·, 2. und 21. XL und 7. XII.

1924191,308

Dänische Dermatologische Gesellschaft. 170. bis 174. Sitzung

(1. XL, 6. XII. 1922, 7. II., 7. III. und 4. IV. 1923) . '. 192 Royal Society of Medicine, London. Sitzungen vom 19. X.,

16. XL und 21. XII. 192272

Royal Society of Medicine, London (Section of Dermatology)

Sitzung vom 19. X. $1922 \quad 310$

Société Française de Dermatologie et de Syphiligraphie.

Sitzungen vom 12. VI., 10. VII. und 13. XL 1924 . 196, 310

Periodische Literatur 77, 198, 315

Buchbesprechungen 243, 357

Personalien und Tagesnachrichten $126,252,362$

Sachregister 363

Namenregister 370 